\title{
Optical Chemo-Sensors for Specific Markers in Transformer Insulating Oil Exploiting Molecularly Imprinted Polymers and Plasmonic Optical Fibers ${ }^{\dagger}$
}

\author{
Letizia De Maria $^{1, *(\mathbb{D} \text {, Francesco Arcadio }}{ }^{2}$, Maria Pesavento ${ }^{3}$, Antonella Profumo ${ }^{3} \mathbb{D}$, Nunzio Cennamo $^{2} \mathbb{D}$ \\ and Luigi Zeni ${ }^{2}$ D \\ 1 RSE S.p.A, Technologies for Transmission and Distribution, Via Rubattino 54, 20134 Milan, Italy \\ 2 Department of Engineering, University of Campania "L. Vanvitelli", Via Roma 29, 81031 Aversa, Italy; \\ Francesco.Arcardio@unicampania.it (F.A.); nunzio.cennamo@unicampania.it (N.C.); \\ luigi.zeni@unicampania.it (L.Z.) \\ 3 Department of Chemistry, University of Pavia, Via Taramelli 12, 27100 Pavia, Italy; \\ maria.pesavento@unipv.it (M.P.); antonella.profumo@unipv.it (A.P.) \\ * Correspondence: letizia.demaria@rse-web.it \\ + Presented at the 2nd International Electronic Conference on Applied Sciences, 15-31 October 2021; \\ Available online: https://asec2021.sciforum.net/.
}

check for updates

Citation: De Maria, L.; Arcadio, F.; Pesavento, M.; Profumo, A.; Cennamo, N.; Zeni, L. Optical Chemo-Sensors for Specific Markers in Transformer Insulating Oil Exploiting Molecularly Imprinted Polymers and Plasmonic Optical Fibers. Eng. Proc. 2021, 11, 13. https://doi.org/10.3390/ ASEC2021-11180

Academic Editor: Nicholas Vassiliou Sarlis

Published: 15 October 2021

Publisher's Note: MDPI stays neutral with regard to jurisdictional claims in published maps and institutional affiliations.

Copyright: (C) 2021 by the authors. Licensee MDPI, Basel, Switzerland. This article is an open access article distributed under the terms and conditions of the Creative Commons Attribution (CC BY) license (https:// creativecommons.org/licenses/by/ $4.0 /)$.

\begin{abstract}
FAL (2-furaldehyde) and furanic derivatives are the main by-products of the thermal degradation of cellulose paper insulation of power transformers' windings. The detection of these compounds in the insulating oil of transformers is essential to investigate the ageing of the oilpaper system in order to avoid failures. To this aim, a non-conventional surface plasmon resonance (SPR) platform in plastic optical fiber (POF) was proposed for the monitoring of a biomimetic receptor specific to detect 2-FAL in transformer oil. In particular, the investigation was performed in mineral oil, which is currently the main insulating liquid for power transformers. A molecularly imprinted polymer (MIP) receptor was used, giving the sensor device a noticeable selectivity and many advantages with respect to the biological counterparts. Furthermore, the study was extended to safer and more environmentally acceptable insulating fluids representing an alternative to mineral oil (i.e., esters). To this aim, the principle and limitations of the SPR chemo-sensor performances have been discussed in this work.
\end{abstract}

Keywords: surface plasmon resonance (SPR); MIP; plastic optical fiber; power transformer

\section{Introduction}

The power transformer is one the most expensive components in the electricity grid. It is an essential asset of the transmission and distribution network, since its reliable operation makes trustworthy the delivery of electricity in the network. Nowadays, power transformers can be exposed to severe operating conditions like irregular stresses or overloads, which can dramatically shorten their useful life. Monitoring power transformer health is a way to enhance their service life and increase power availability $[1,2]$. Some improvements in predictive maintenance and cost-saving, for this electrical equipment, are achievable by the widespread integration of new sensors and devices, especially those based on optical fibres-based technologies. Due to their low invasiveness and the dielectric nature of the optical fibers, these sensors could be effective for monitoring different physical and chemical parameters in the presence of high electromagnetic interferences as in the transmission and distribution network. An extensive review of the potential applications of fiber optic sensors in power transformers is presented in [2]. In particular, for oil-filled transformers, the frequent control of chemical markers in the insulating oil could provide an early warning of incipient failures or accelerated ageing of dielectric parts. In this framework, a surface plasmon resonance plastic optical fiber sensor, combined with specific 
molecularly imprinted polymers (MIPs), was reported by the authors in [3,4] as a diagnostic tool for chemical markers detection in transformers. In particular, dibenzyl disulfide (DBDS), which is correlated to the presence of corrosive sulfur in insulating mineral oil and to the formation of conductive deposits inside the insulating paper of the windings (copper sulfide) [5], and furfuraldehyde (2-FAL), which is a by-product of the thermal degradation of the paper insulation (paper windings, pressboards) in transformers $[6,7]$ have been considered as significant markers. Because of the particular nature of the liquid in which the two markers must be determined, i.e., the transformer oil, bioreceptors as antibodies or aptamers were not considered due to the well-known characteristic of the biomolecules to better perform in aqueous media. Instead, a synthetic receptor as an MIP was applied, which has the additional advantages of being more easily developed and much less expensive than bioreceptors. For these reasons, MIPs are particularly suitable for implementation of low-cost sensing devices. In our case, they have been implemented in a plastic optical fibre, which acts as an optical platform for the synthetic receptor and makes signal transduction based on the surface plasmon resonance phenomenon possible.

As a proof of concept, this SPR sensor, combined with MIPs (POF-SPR-MIP), was used for the detection of two important markers of degradation of the solid insulating system of transformers by analysing the mineral oil, which is currently the main insulating liquid for power transformer.

In the last few decades, the new requirements of oil filled transformers with safer performances and with lower impact on the environment are pushing for the use of fluids alternative to mineral oil, like synthetic and natural esters. Esters, which consist of triglycerides or esters of fatty acids, present excellent performances in terms of biodegradability and fire points [8] and some benefits in terms of prolonging the life of the transformers' solid insulating system (that is, the oil-paper insulation). According to the state-of-art on oil-paper degradation phenomena, some physical and chemical properties of esters, like hygroscopic features, acidity, etc., could influence the nature or concentration of the chemical markers representing the by-products of transformers' oil-paper system ageing [9]. From a diagnostic point of view, the use of a new matrix in which the detection of the chemical markers should be performed can influence the optical response of the POF-SPRMIP sensors. In this work, we focus on some features of the optical platform and on the MIP layer of the proposed POF-SPR-MIP sensor, in view of an extension of the chemical markers detection in the new ester-based insulating oil matrix. We focalized on the 2-FAL detection since the presence of antioxidants, as DBDS, is highly unlikely in ester oils [10].

\section{SPR Chemo-Sensor System Based on MIP Receptor}

As reported in previous works of the same authors [4,5], the optical chemo-sensor named POF-SPR-MIP is achieved through a combination of the SPR platform, manufactured in a plastic optical fiber, and a synthetic receptor, MIP, synthesized by molecular imprinting methods. An outline of the experimental configuration used for the measurements is reported in Figure 1.

The measurement apparatus consists of a halogen lamp and a spectrometer connected to a PC. The white light source presents an emission range from $360 \mathrm{~nm}$ to $1700 \mathrm{~nm}$, whereas the spectrometer has a detection range from $350 \mathrm{~nm}$ to $1000 \mathrm{~nm}$. The transmission spectra and data values are displayed online on a computer screen and saved by proprietary software.

The use of a relatively thick MIP layer provides several advantages with respect to other bio- and chemical receptors, as for example antibodies, guaranteeing high stability in an aggressive matrix, like the mineral oil, while maintaining high selectivity and sensitivity. Figure 1 shows a cross-section of the POF-SPR-MIP sensor. 


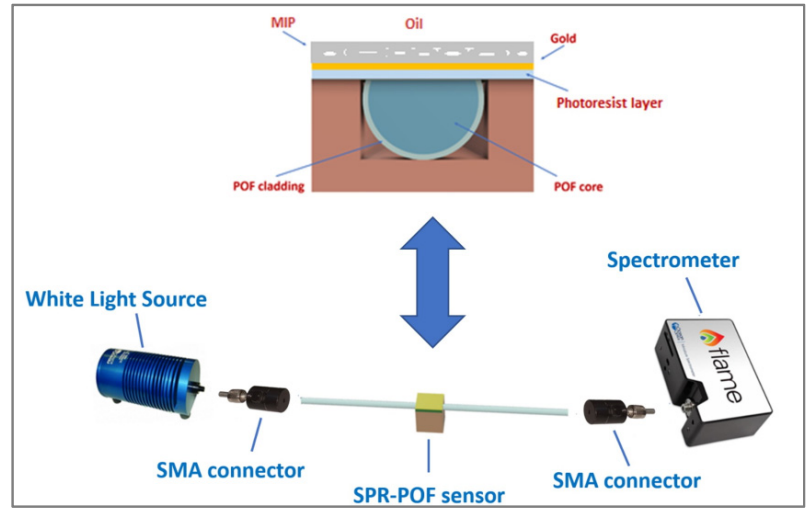

Figure 1. Scheme of the experimental setup and cross-section of the POF-SPR-MIP sensor.

\section{MIP Layer}

With respect to more standard molecular recognition elements (MRE), MIPs represent a new emerging class increasingly employed to realize biosensors. Up to now, very few examples have been reported in the literature of the coupling between MIPs and POFs to realize biomimetic optical sensors [11-13], a number of which came from our group. MIPs are synthetic solids with many favorable characteristics with respect to bio-receptors, such as an easier and faster preparation, the possibility of application outside the laboratory, for example in real environment conditions, and in non-aqueous solutions, and a longer duration [14]. They are porous solids containing specific sites interacting with the molecule of interest, according to a "key and lock" model. In the case of MIPs, the receptor site is a "cavity" or "pocket" which is formed in the polymeric structure by using the molecular imprinting techniques. They consist of the polymerization of the aggregates formed between the molecule of interest (which is the template) and a suitable functional monomer. The principle of the preparation of a specific MIP is briefly reported in the Scheme of Figure 2.

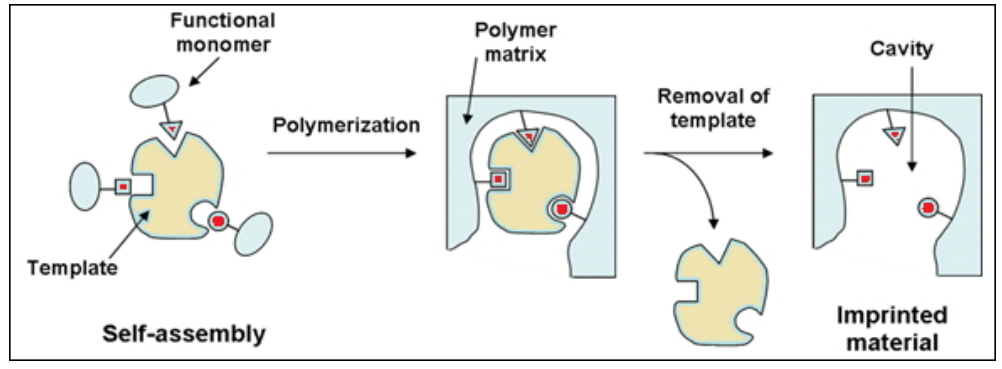

Figure 2. Principle of the preparation of a specific MIP.

In the sensors here examined, the MIP is obtained by radical polymerization of a mixture of template (2-FAL, 1 mole): functional monomer (methacrylic acid, 4 moles): cross-linker (divinylbenzene, 40 moles). This liquid is called the pre-polymeric mixture. The mixture is simply deposited over gold, and spun in order to reduce and homogenize the amount of polymer, which will be successively formed by radical polymerization at high temperatures $\left(72{ }^{\circ} \mathrm{C}\right)$.

In this last decade, different MIPs have been specialized for proving the high versatility of this kind of platform in matrices with a refractive index higher than water. Water is a wellsuited solvent for measurements with the platforms here described since its refractive index (RI) matches the operative range of the proposed SPR sensors (1.33-1.42) [14]. Problems can arise when liquid samples with higher RI are considered. In that case, an elegant solution to the problem can be the use of an MIP layer with RI matching the useful range, and sufficiently thick as to shield the effect of the overlaying liquid, as explained in the following section. 


\section{Plasmonic Response of the POF-SPR-MIP Sensor System}

As an example, typical spectra of a POF-SPR-MIP sensor in different media (bulk) are reported in Figure 3. The pristine transmission spectrum is normalized to the corresponding spectrum of the platform without an MIP layer (bare surface of the sensor) in air.

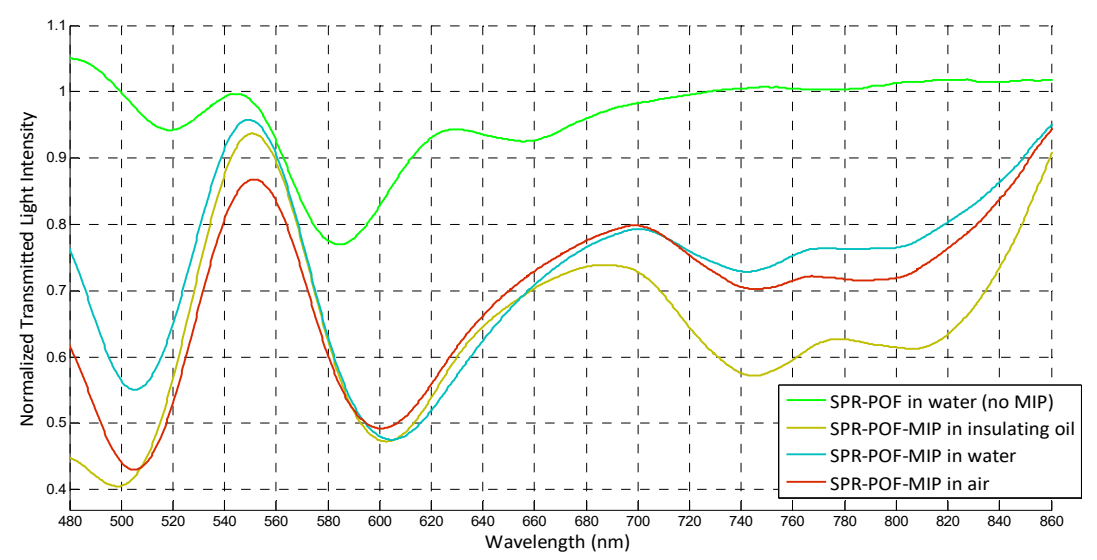

Figure 3. SPR spectra of a POF-SPR-MIP sensor in different media: air, water, and mineral oil. For comparison, the SPR spectrum of the bare surface in contact with water (without an MIP layer) is also reported.

The spectra of the POF-SPR-MIP in media with largely different refractive index, i.e., air, water, and mineral oil are very similar, different from that of the bare surface (without MIP) in water. As shown in Figure 3, the peak at about $740 \mathrm{~nm}$ is similar in air and in insulating oil, indicating that the plasmonic resonance does not depend on the RI of the overlaying medium (bulk solution) but only on the presence of the specific chemical marker.

The resonance, in fact, shifts to higher wavelengths when the sensor is contacted with bulk solutions containing different concentrations of 2-FAL [4]. This gives information about the affinity of the MIP for the molecule of interest. In the considered case, the affinity, measured as the equilibrium constant of the adsorption equilibrium $\mathrm{K}_{\mathrm{aff}}$, is at about $10^{6} \mathrm{M}^{-1}$, corresponding to a lower detection range of $\sim 10^{-6} \mathrm{M}$. This lower detection limit could be suitable for the detection of the 2-FAL marker in a different insulating oil matrix (ester) for a warning on accelerated ageing of the transformer.

With previous investigations, we have demonstrated the optical response of the POFSPR-MIP for 2-FAL in different case studies, i.e., in exhausted oil samples spilled from two ex-serviced current transformers used as a bench test [4,15], in mineral oil samples, representative of the solid insulating system of transformers, exposed to controlled thermal treatment to simulate aging of the insulating paper $[16,17]$. In these cases, the likely presence of impurities did not induce any shift of resonance wavelengths in the SPR spectra, indicating that they are not adsorbed at the imprinted sites. This is another favorable aspects for extending this application to a new insulating matrix.

\section{Conclusions}

Molecularly imprinted polymers represent a key solution for the development of SPR optical fiber chemo-sensor for detecting transformers' oil degradation. Results of previous investigations confirmed that the high sensitivity of the developed POF-SPR-MIP sensors and the high specificity of the MIP layer are the main strengths allowing to face the new challenging application of these sensors to a different insulating matrix (i.e., ester oil).

Author Contributions: Conceptualization, validation, writing—original draft, L.D.M., M.P., F.A. and N.C.; writing—review \& editing, L.D.M., L.Z., M.P. and A.P. All authors have read and agreed to the published version of the manuscript. 
Funding: This work was financed by the Research Fund for the Italian Electrical System under the Contract Agreement between RSE and the Ministry of Economic Development-General Directorate for Energy and Mining Resources stipulated on 29 July 2009 in compliance with the Decree of 19 March 2009.

Institutional Review Board Statement: Not applicable.

Informed Consent Statement: Not applicable.

Data Availability Statement: Not applicable.

Conflicts of Interest: The authors declare no conflict of interest.

\section{References}

1. Chakravorti, S.; Dey, D.; Chatterjee, B. Recent Trends in the Condition Monitoring of Transformers: Theory, Implementation and Analysis; Springer: London, UK, 2013.

2. N'cho, J.S.; Fofana, I. Review of fiber optic diagnostic techniques used in power transformers. Energies 2020, 13, 1789. [CrossRef]

3. Cennamo, N.; De Maria, L.; D'Agostino, G.; Zeni, L.; Pesavento, M. Monitoring of Low Levels of Furfural in Power Transformer Oil with a Sensor System Based on a POF-MIP Platform. Sensors 2015, 15, 8499-8511. [CrossRef] [PubMed]

4. Cennamo, N.; De Maria, L.; Chemelli, C.; Profumo, A.; Zeni, L.; Pesavento, M. Markers detection in transformer oil by plasmonic chemical sensor system based on POF and MIPs. IEEE Sens. J. 2016, 16, 7663-7670. [CrossRef]

5. Scatiggio, F.; Tumiatti, V.; Maina, R.; Pompili, M.; Bartnikas, R. Corrosive Sulfur in nsulating Oils: Its Detection and Correlated Power Apparatus Failures. IEEE Trans. Power Deliv. 2008, 23, 508-509. [CrossRef]

6. Lundgaard, L.E.; Hansen, W.; Linhjell, D.; Painter, T.J. Aging of oil-impregnated paper in power transformers. IEEE Trans. Power Deliv. 2004, 19, 230-239. [CrossRef]

7. N'cho, J.S.; Fofana, I.; Hadjadj, Y.; Beroual, A. Review of Physicochemical-Based Diagnostic Techniques for Assessing Insulation Condition in Aged Transformers. Energies 2016, 9, 367. [CrossRef]

8. Scatiggio, F.; Pompili, M. Evaluation of vegetable ester for filling large power transformers. In Proceedings of the 2016 IEEE International Conference on Dielectrics (ICD), Montpellier, France, 3-7 July 2016; pp. 1052-1056. [CrossRef]

9. Rozga, P.; Beroual, A.; Przybylek, P.; Jaroszewski, M.; Strzelecki, K. A Review on Synthetic Ester Liquids for Transformer Applications. Energies 2020, 13, 6429. [CrossRef]

10. Scatiggio, F.; Rebolini, M. Preliminary Study for Use of Vegetable Esters in Big Power Transformers; Paper D1-202; CIGRE: Paris, France, 2014.

11. Pesavento, M.; Marchetti, S.; De Maria, L.; Zeni, L.; Cennamo, N. Sensing by Molecularly Imprinted Polymer: Evaluation of the Binding Properties with Different Techniques. Sensors 2019, 19, 1344. [CrossRef] [PubMed]

12. Uzun, L.; Turner, A.P.F. Molecularly-imprinted polymers sensors: Realising their potential. Biosens. Bioelectron. 2016, 76, 131-144 [CrossRef] [PubMed]

13. Haupt, K.; Mosbach, K. Molecularly imprinted polymers and their use in biomimetic sensors. Chem. Rev. 2000, 100, 2495-2504. [CrossRef] [PubMed]

14. Cennamo, N.; Pesavento, M.; Zeni, L. A review on simple and highly sensitive plastic optical fiber probes for bio-chemical sensing Sens. Actuators B Chem. 2021, 331, 129393. [CrossRef]

15. De Maria, L.; Scatiggio, F.; Pesavento, M.; Cennamo, N.; Zeni, L. Toward an optical monitoring of chemical markers in transformers insulating oil. In Proceedings of the 2019 IEEE 20th International Conference on Dielectric Liquids (ICDL), Roma, Italy, 23-27 June 2019; pp. 1-4. [CrossRef]

16. De Maria, L.; Borghetto, J.; Cennamo, N.; Scatiggio, F.; Pesavento, M.; Zeni, L. Frequency dielectric spectroscopy and an innovative optical sensor to assess oil-paper degradation. IEEE Trans. Dielectr. Electr. Insul. 2020, 27, 1728-1735. [CrossRef]

17. De Maria, L.; Cice, L.; Bartalesi, D.; Borghetto, J.; Tavakoli, A.; Scatiggio, F.; Gasparini, D.; Pesavento, M. An optical SPR sensor for monitoring accelerated ageing of oil-paper insulation of transformers. In Sensors and Microsystems; Lecture Notes in Electrical Engineering; Springer: Cham, Switzerland, 2019; Volume 629. 